\title{
The high-dose dexamethasone suppression test is inferior to pituitary dynamic enhanced MRI in the differential diagnosis of ACTH-dependent Cushing's syndrome
}

\section{Xiaoli Shi}

Tongji Hospital of Tongji Medical College of Huazhong University of Science and Technology

\section{Tingting Du}

Tongji Hospital of Tongji Medical College of Huazhong University of Science and Technology

\section{Dan Zhu}

Tongji Hospital of Tongji Medical College of Huazhong University of Science and Technology

Delin Ma

Tongji Hospital of Tongji Medical College of Huazhong University of Science and Technology Kun Dong

Tongji Hospital of Tongji Medical College of Huazhong University of Science and Technology

\section{Xuemin Peng}

Tongji Hospital of Tongji Medical College of Huazhong University of Science and Technology

Jiaojiao Huang

Tongji Hospital of Tongji Medical College of Huazhong University of Science and Technology

\section{Ting Lei}

Tongji Hospital of Tongji Medical College of Huazhong University of Science and Technology

\section{Xue feng Yu}

Tongji Hospital of Tongji Medical College of Huazhong University of Science and Technology

\section{Juan Chen}

Tongji Hospital of Tongji Medical College of Huazhong University of Science and Technology

Yan Yang ( $\triangle$ yangyan6910@163.com )

Tongji Hospital of Tongji Medical College of Huazhong University of Science and Technology https://orcid.org/0000-0001-8146-8040

\section{Research Article}

Keywords: Cushing's syndrome, pituitary dynamic enhanced MRI, HDDST

Posted Date: February 24th, 2021 
DOI: https://doi.org/10.21203/rs.3.rs-230624/v1

License: (c) (1) This work is licensed under a Creative Commons Attribution 4.0 International License. Read Full License

Version of Record: A version of this preprint was published at Endocrine on October 4th, 2021. See the published version at https://doi.org/10.1007/s12020-021-02891-y. 


\section{Abstract \\ Purpose}

The differential diagnosis of ACTH-dependent Cushing's syndrome remains a challenge in clinical practice. The aim of the present study was to assess the diagnostic performance of pituitary dynamic enhanced MRI(dMRI), high-dose dexamethasone suppression test $\square \mathrm{HDDST} \square$ and combination of both tests in patients with ACTH-dependent Cushing's syndrome.

\section{Methods}

119 consecutive cases with surgery confirmed ACTH-dependent Cushing's syndrome were enrolled: 101 patients with proven Cushing's disease and 18 patients with proven ectopic ACTH syndrome. All patients underwent pituitary dMRI and HDDST. The sensitivity and the specificity of pituitary dMRI, HDDST, and combination of both tests were calculated.

\section{Results}

The sensitivity and specificity of the pituitary dMRI for the diagnosis of Cushing's disease was $80.2 \%$ and $83.3 \%$ respectively, and the positive predictive value was $96.4 \%$. The sensitivity and specificity of HDDST was $70.3 \%$ and $77.8 \%$, and the positive predictive value was $94.7 \%$. Regarding the combination of both tests, the combined criteria of a more than $50 \%$ suppression of serum cortisol in HDDST and a positive finding in pituitary dMRI had given a high specificity of $94.4 \%$ and a sensitivity of $59.4 \%$. The combined criteria of a greater than $68 \%$ suppression in HDDST and/or a positive finding in pituitary dMRI, provide a sensitivity of $86.1 \%$ and a specificity of $83.3 \%$.

\section{Conclusions}

Pituitary dMRI was superior to HDDST in the differential diagnosis of ACTH-dependent Cushing's syndrome. HDDST was recommended to combined with pituitary dMRI in the diagnosis process because of the significantly increased specificity of the combination.

\section{Introduction}

Cushing's syndrome (CS) is a clinical state caused by adrenal gland secreting excessive glucocorticoid. It can be divided into adrenocorticotropic hormone (ACTH)-dependent Cushing's syndrome and ACTH independent Cushing's syndrome. ACTH-dependent Cushing syndrome is caused by tumors that produce ACTH. The two main types of ACTH-dependent Cushing's syndrome are Cushing's disease (CD) caused by pituitary adenomas and ectopic ACTH syndrome (EAS) resulted from ACTH secreting tumors outside the pituitary, such as thymic carcinoid, bronchial carcinoid and medullary. 
The diagnosis of ACTH-dependent Cushing's syndrome could be a challenge in clinical practice. The core of the problem is how to distinguish between an ACTH-producing pituitary microadenoma and an extrapituitary source of ACTH secretion. Bilateral inferior petrosal sinus sampling (BIPSS) is an accurate method with high sensitivity and specificity to distinguish CD and EAS[1]. However, this technology is expensive and invasive. Therefore, the noninvasive examinations play an important role in clinical practice.

HDDST has been the most commonly used biochemical tool for differential diagnosis ACTH-dependent CS for many years. A positive response is a decrease in plasma cortisol to $50 \%$ or less of the baseline level. The HDDST is mostly positive in patients with CD and negative in patients with EAS. However, some patients with severe $C D$, especially those with large pituitary tumors and high cortisol levels, may show a negative response, while nearly $10 \%$ of patients with EAS show a positive response [2].

Magnetic resonance imaging (MRI) was the primary modality used to detect pituitary tumors. According to reports, nearly $50 \%$ of patients have false negative results in conventional pituitary MRI [3], which limited its value in the diagnosis of CD. Compared with conventional MRI, dynamic-enhanced MRI (dMRI), a method of dynamically acquiring T1-weighted MRI scans after intravenous injection of contrast agents, can provide more information about pituitary adenomas[4], which potentially improves the sensitivity of pituitary tumor detection, but it may reduce the specificity of detection [5].

The aim of the present study was to assess the diagnostic performance of pituitary dMRI, HDDST and the combination of both tests in a consecutive series of patients with ACTH-dependent CS.

\section{Methods}

\section{Participants}

For this retrospective study, we enrolled a consecutive series of patients with ACTH-dependent Cushing's syndrome between 2008 and 2020 at our hospital, including patients diagnosed as Cushing's disease and patients diagnosed as ectopic ACTH syndrome. Clinical, laboratory, radiological, pathological, treatment, and outcome data were obtained from the hospital records of all patients. Patients who didn't undergo pituitary dMRI, lack HDDST or receive any drug known to affect the hypothalamic-pituitaryadrenal axis were excluded from the retrospective study. The diagnosis of CS was made on the basis of the criteria in the Endocrine Society Clinical Practice Guideline[6]. In all cases, the diagnosis was confirmed by a pathological examination or by clinical remission after the operation. The written informed consent was obtained from all patients that enrolled.

\section{High-dose dexamethasone test (HDDST).}

Before the test, blood samples were collected at 8 am, and plasma cortisol levels were measured and recorded as base level. Then, $2 \mathrm{mg}$ of dexamethasone was taken orally every 6 hours for 2 days, and plasma cortisol levels were measured at $8 \mathrm{am}$ in the next morning. A positive response is that the level of 
plasma cortisol after taking the dexamethasone is suppressed to less than half of baseline level in HDDST. A positive response in the HDDST was considered to be consistent with CD.

\section{Imaging}

Pituitary dMRI was performed on a 3.0 T MR scanner. The significantly enhanced hypointensity inside the pituitary gland is a reliable sign for the diagnosis of pituitary adenoma. In the early stage of dynamic cycles, pituitary adenoma showed a discrete hypointensity, which gradually enhanced with time delay. Two experienced radiologists independently performed the imaging interpretation. They knew the patient's clinical information but were blinded to the surgical and pathological results. If there was a disagreement, the scan results should be reviewed and evaluated by two radiologists by consensus. The radiological interpretations of pituitary dMRI were classified as positive, questionable, or negative. When the result was found to be negative or doubtful, the pituitary dMRI was classified as negative for statistical analysis.

\section{Laboratory assays}

Serum cortisol was measured by chemiluminescent enzyme immunoassay. Plasma ACTH levels were measured by immunoradiometric assay.

\section{Treatment and pathology}

Transsphenoidal surgery was performed on patients diagnosed with CD. Resection of ectopic ACTH secreting tumor was performed on EAS patients. The diagnosis was confirmed by pathology indicating an ACTH immunohistological staining positive tumor or clinical remission after the operation. Clinical remission refers to serum cortisol level decreased to the normal range or lower than the normal range.

\section{Statistical Analysis}

Continuous variables were expressed as mean $\pm S D$, median and interquartile range. The sensitivity, specificity, accuracy, false-positive ratio, and false-negative ratio, positive predictive value, negative predictive value of dMRI, HDDST and combination of both tests were calculated by comparing the results of each diagnostic procedure with gold standard. The gold standard was pathological examination or clinical remission after the operation. Receiver operating characteristic (ROC) curve was also used to assess value of dMRI, HDDST and combination of both tests for diagnosis. The area under the curve (AUC) was calculated and the best cut-off values were define. The point on a ROC curve offering highest specificity and sensitivity provides the best cut-off value. Statistical analysis was performed using SPSS 24.0 software.

\section{Results}

\section{Demographic and Clinical profile}


The study included a total of 119 patients with ACTH-dependent CS, including 66 (84.9\%) patients with CD and 18 (15.1\%) patients with EAS. Demographic data showed that of the 119 CS patients, 27 (22.7\%) were male and 92 (77.3\%) were female. The age range of the patients was 13 to 67 years and the average age was $38.2 \pm 12.5$ years. The median midnight (23:00) plasma cortisol level was $23.7(18.0,32.7) \mu \mathrm{g} / \mathrm{dL}$ and median ACTH level was $24.3(13.8,40.8) \mathrm{pmol} / \mathrm{L}$ (normal range 1.6-13.9 pmol/L). All the patients underwent HDDST and pituitary dMRI.

\section{Pituitary dMRI in the differential diagnosis of ACTH-dependent CS}

84 of 119 patients had definitely positive findings in pituitary dMRI, of whom, 16 were macroadenoma and 67 were microadenoma. 2 of the 84 patients were definitely diagnosed with EAS. one patient underwent transsphenoidal surgery but had no clinical remission after surgery, and she was found to be EAS at subsequent follow-up. Thus, totally, in 3 of 84 dMRI-positive patients, an ectopic source of ACTH was found. The remaining 81 patients underwent transsphenoidal surgery. Among these 81 patients, 73 were pathologically confirmed to be ACTH-positive pituitary adenomas. 8 of the 81 patients were found to have pituitary tumor, but the histological results showed negative ACTH staining. They were cured by surgery and were thus diagnosed with Cushing's disease.

14 patients had questionable findings and 21 patients had negative findings in pituitary dMRI. These 35 patients were all classified as negative for statistical analysis. Among them, 13 patients were definitely diagnosed with EAS and thus didn't undergo transsphenoidal surgery. 22 of these 35 patients were referred for transsphenoidal surgery based on HDDST or IPSS results. 2 of the 22 patients were not cured by surgery and they were finally found to be EAS at subsequent follow-up. In the remaining 20 patients (12 with negative dMRI, 8 with inconclusive dMRI), a pituitary source of ACTH was proven. 17 of the 20 patients were pathologically confirmed to have pituitary ACTH tumors. In the other 3 patients, no tumor was found, but complete cure or obvious remission after surgery proved the presence of a pituitary source of ACTH. (Table 1).

The sensitivity and specificity of pituitary dMRI to detect a pituitary source of ACTH was $80.2 \%$ and $83.3 \%$ respectively, and the accuracy was $80.3 \%$. The positive and negative predictive values were $96.4 \%$ and $42.9 \%$, respectively. The false negative ratio and the false positive ratio were $19.8 \%$ and $16.7 \%$ respectively. Table 2 showed the diagnostic performance figures of pituitary dMRI for the diagnosis of CD.

\section{HDDST in the differential diagnosis of ACTH-dependent CS}

71 cases out of 101 cases with Cusing's disease showed a positive response in the HDDST. 4 cases out of 18 cases of EAS showed a positive response in the HDDST (Table 1). Here, a positive response is that the level of plasma cortisol after taking the dexamethasone is suppressed to less than half of baseline level in HDDST. 
The sensitivity of HDDST for the diagnosis of CD was $70.3 \%$, whereas specificity was $77.8 \%$, and the accuracy was $71.4 \%$. The negative and positive predictive values were $31.8 .6 \%$ and $94.7 \%$ respectively, and the false-negative and false-positive ratios were $29.7 \%$ and $22.2 \%$, respectively (Table 2 ).

\section{Combined pituitary dMRI and HDDST in the differential diagnosis of ACTH-dependent CS}

There were 61 patients having definitely positive findings in pituitary dMRI and positive responses in HDDST, of whom 60 patients were CD and 1 patient was EAS. The remaining 58 patients had either negative findings in pituitary dMRI or unsuppressed cortisol levels in HDDST, of whom 41 patients were CD and 17 patients were EAS (Table 1). Hence, the combined test carried a sensitivity of $59.4 \%$ for the diagnosis of CD, whereas specificity was $94.4 \%$, and accuracy was $64.7 \%$. The negative and positive predictive values were $29.3 \%$ and $98.4 \%$ respectively, and the false-negative ratio and false-positive ratio were $40.6 \%$ and $5.6 \%$, respectively (Table 2 ).

There were 98 patients had either positive findings in pituitary dMRI or unsuppressed cortisol levels in HDDST, of whom 92 patients were CD and 6 patients were EAS. The remaining 21 patients having negative findings in pituitary dMRI and negative responses in HDDST, of whom 9 patients were $\mathrm{CD}$ and 12 patients was EAS (Table 1). Hence, the combined test carried a sensitivity of $91.1 \%$ for the diagnosis of $C D$, whereas specificity was $66.7 \%$, and accuracy was $87.4 \%$. The negative and positive predictive values were $57.1 \%$ and $93.9 \%$ respectively, and the false-negative ratio and false-positive ratio were $8.9 \%$ and $33.3 \%$, respectively (Table 2 ).

\section{ROC contrast analyses among HDDST, dMRI and HDDST+dMRI}

Although, a $50 \%$ suppression of serum cortisol after HDDST is a widely used cutoff, to avoid the bias of predetermined criteria, ROCs were constructed for HDDST and dMRI for the differential diagnosis between CD and EAS. We then calculated a ROC-based cutoff for HDDST. As shown in Figure 1, the area under curve (AUC) of HDDST was $0.809(95 \% \mathrm{Cl}, 0.714-0.903 ; \mathrm{P}<0.001)$. The optimal cut-off value was a greater than $42.5 \%$ suppression, which had a sensitivity of $76.2 \%$ and a specificity of $77.8 \%$ in the diagnosis of CD. The AUC of dMRI was $0.818(95 \% \mathrm{Cl}, 0.709-0.927 ; \mathrm{P}<0.001)$, showing that dMRI was slightly superior to HDDST alone in the differential diagnosis of ACTH-dependent CS.

To further determine whether the combination of HDDST and dMRI achieved a higher diagnostic accuracy, we also constructed a ROC curve for HDDST+dMRI (Figure 1). The AUC of HDDST+dMRI was $0.875(95 \% \mathrm{Cl}, 0.779-0.971 ; \mathrm{P}<0.001)$. The optimal cut-off value was a greater than $68 \%$ suppression of serum cortisol after HDDST and/or a positive finding in pituitary dMRI, which had a sensitivity of $86.1 \%$ and a specificity of $83.3 \%$ in the diagnosis of CD. Thus, the combination of HDDST and AMRI achieved a higher diagnostic accuracy.

\section{Discussion}


The differentiation between $C D$ and EAS remains one of the most challenging issues for the clinical endocrinologist. In the current study, we investigated the noninvasive tests (pituitary dMRI and HDDST) in the differential diagnosis of ACTH-dependent CS. The present study showed that both the specificity and sensitivity of pituitary dMRI were higher than that of HDDST. When combined HDDST with pituitary dMRI, it provides a higher diagnostic accuracy then HDDST or dMRI alone in the differential diagnosis of ACTHdependent CS.

MRI is the best imaging tool for the detection of pituitary tumors. However, the low sensitivity of conventional pituitary MRI limited its value in the differential diagnosis of Cushing's syndrome. The sensitivity of conventional pituitary MRI for detecting ACTH-secreting pituitary adenomas varies greatly in literatures, and it is generally believed that about $30-50 \%$ of pituitary adenomas are not correctly identified[7-9]. With the development of MRI technology, the detection rate of pituitary adenomas has been improved significantly. Compared with conventional MRI, pituitary dMRI can obtain repeated images within a few seconds after the injection of contrast agent, providing more information about pituitary adenomas. It is usually considered that although pituitary dMRI improves the sensitivity of pituitary adenoma detection, it is associated with an increased false-positive ratio [10]. The sensitivity and false negative ratio of pituitary dMRI in our study were $80.2 \%$ and $19.8 \%$ respectively. This indicated that in only less than $20 \%$ of patients with CD, pituitary dMRI failed to show the presence of pituitary adenoma. The false negative results are partly due to the small size of the pituitary adenoma, or because the lesions have similar signal and enhancement features with normal pituitary. It is worth noting that in 3 cases with false negative dMRI findings, the presence of ACTH secreting adenoma also could not be confirmed by surgical exploration and pathological examination. For these patients, the final diagnosis of CD was clear because hypercortisolemia has been completely cured after surgery. Consistent with our results, other studies have also reported a complete cure of elevated corticosteroids after hypophysectomy, but there is no histological evidence of tumor $[3,11,12]$. These observations suggested that a part of pituitary ACTH lesions were histologically silent. The specificity and false positives ratio of pituitary dMRI in our study were calculated as $83.3 \%$ and $16.7 \%$, respectively. The specificity of pituitary dMRI was widely explored, ranging from 62 to $84 \%$ as previously reported $[5,13,10]$. False positives mainly result from the incidence of pituitary asymptomatic microadenomas or technical artefacts. This suggests that the pituitary lesions detected on dMRI may be coincidental and sometimes may lead to unnecessary pituitary resection, so it needs to be confirmed by other complementary diagnostic procedures.

The HDDST is still in common use as a method in differential diagnosis of ACTH-dependent Cushing's syndrome. Although the accuracy of this test may be compromised by various factors, the major problem with HDDST is its diagnostic inaccuracy [14-16]. In all cases, the response is related to the original cortisol secretion rate: in patients with lower basal cortisol levels, greater suppression is usually observed. Some patients with severe CD, especially those with large tumors and extremely high levels of cortisol, may lack response, while at least $10 \%$ of patients with EAS have a positive response[2]. The significant overlap in responses of CD and EAS to HDDST compromises the diagnostic accuracy of this test. According to David C. Aron et al, the sensitivity of HDDST for the diagnosis of CD was $81.0 \%$ and the specificity was only $66.7 \%$ [14]. A more recent study evaluated a total of 85 patients with CD and 10 
patients with EAS. The sensitivity and specificity of HDDST were $79 \%$ and $80 \%$, respectively[17]. Although HDDST was widely used, different sensitivity and specificity have been reported in different series. The number of patients with EAS in series may be a factor affecting the assessment. In our study, using a generally accepted cutoff of $50 \%$ suppression, the sensitivity and specificity of HDDST were $70.3 \%$ and $77.8 \%$, respectively. To avoid the bias of predetermined criteria, we constructed a ROC for HDDST and found that more than $42.5 \%$ suppression of cortisol levels had the highest accuracy, with a sensitivity of $76.2 \%$ and a specificity of $77.8 \%$. The diagnostic accuracy is still lower than that of dMRI. Thus, our data also supported that HDDST shows a poor diagnostic performance and thus cannot establish the etiological differential diagnosis by itself alone.

Although the diagnostic performance of HDDST alone was not good enough, the combination of pituitary dMRI and HDDST(both positive) achieved a high specificity of $94.4 \%$ and positive predictive value of $98.4 \%$ in distinguishing between patients with CD from those with EAS. Moreover, the combination of both tests in the present study showed a specificity similar to the BIPSS according to previous literatures [18-21]. The data presented here suggested that patients with a positive finding in pituitary dMRI and a positive response in HDDST do not require further BIPSS to confirm the diagnosis of CD. Further, we found that the combined criteria of a greater than $68 \%$ suppression in HDDST, and/or a positive finding in pituitary dMRI, provide a sensitivity of $86.1 \%$ and a specificity of $83.3 \%$, which was also superior to HDDST or dMRI alone. However, it is worth to note that the diagnostic accuracy is based on the present series. So, it requires validation to apply this criteria in clinical.

The limitation of the study is the possibility of selection bias. We excluded patients who did not undergo surgery. Moreover, a considerable number of EAS patients may be excluded because they cannot be confirmed by surgery and pathology.

In summary, pituitary dMRI was superior to HDDST in the differential diagnosis of ACTH-dependent Cushing's syndrome. Although the diagnostic performance of HDDST alone was unsatisfactory, the combination of HDDST and pituitary dMRI achieved high specificity. Therefore, HDDST was recommended to combined with pituitary dMRI in the diagnosis process.

\section{Abbreviations}

CS = Cushing's syndrome, ACTH = adrenocorticotropic hormone, $C D=$ Cushing's disease, EAS = ectopic ACTH syndrome, dMRI = dynamic enhanced MRI, HDDST = high-dose dexamethasone suppression test, BIPSS = Bilateral inferior petrosal sinus sampling.

\section{Declarations}

\section{Compliance with Ethical Standards}

Funding This study was supported by grants from the National Natural Science Foundation of China (No. 81800686) and the Science and Technology Program of Hubei Province (No. 2019CFB316). 
Conflict of interest The authors have no conflicts of interest to disclose.

Ethical approval All procedures performed in studies involving human participants were in accordance with the ethical standards of the institutional and/or national research committee and with the 1964 Helsinki declaration and its later amendments or comparable ethical standards.

Informed consent The written informed consent was obtained from all patients that enrolled.

\section{References}

1. Pecori Giraldi, F., Cavallo, L.M., Tortora, F., Pivonello, R., Colao, A., Cappabianca, P., Mantero, F.: The role of inferior petrosal sinus sampling in ACTH-dependent Cushing's syndrome: review and joint opinion statement by members of the Italian Society for Endocrinology, Italian Society for Neurosurgery, and Italian Society for Neuroradiology. Neurosurgical focus 38(2), E5 (2015). doi:10.3171/2014.11.Focus14766

2. Nishioka, H., Yamada, S.: Cushing's Disease. Journal of clinical medicine 8(11) (2019). doi:10.3390/jcm8111951

3. Kaskarelis, I.S., Tsatalou, E.G., Benakis, S.V., Malagari, K., Komninos, I., Vassiliadi, D., Tsagarakis, S., Thalassinos, N.: Bilateral inferior petrosal sinuses sampling in the routine investigation of Cushing's syndrome: a comparison with MRI. AJR. American journal of roentgenology 187(2), 562-570 (2006). doi:10.2214/ajr.05.0557

4. Vitale, G., Tortora, F., Baldelli, R., Cocchiara, F., Paragliola, R.M., Sbardella, E., Simeoli, C., Caranci, F., Pivonello, R., Colao, A.: Pituitary magnetic resonance imaging in Cushing's disease. Endocrine 55(3), 691-696 (2017). doi:10.1007/s12020-016-1038-y

5. Friedman, T.C., Zuckerbraun, E., Lee, M.L., Kabil, M.S., Shahinian, H.: Dynamic pituitary MRI has high sensitivity and specificity for the diagnosis of mild Cushing's syndrome and should be part of the initial workup. Hormone and metabolic research $=$ Hormon- und Stoffwechselforschung $=$ Hormones et metabolisme 39(6), 451-456 (2007). doi:10.1055/s-2007-980192

6. Nieman, L.K., Biller, B.M., Findling, J.W., Newell-Price, J., Savage, M.O., Stewart, P.M., Montori, V.M.: The diagnosis of Cushing's syndrome: an Endocrine Society Clinical Practice Guideline. The Journal of clinical endocrinology and metabolism 93(5), 1526-1540 (2008). doi:10.1210/jc.2008-0125

7. Boscaro, M., Arnaldi, G.: Approach to the patient with possible Cushing's syndrome. The Journal of clinical endocrinology and metabolism 94(9), 3121-3131 (2009). doi:10.1210/jc.2009-0612

8. Colombo, N., Loli, P., Vignati, F., Scialfa, G.: MR of corticotropin-secreting pituitary microadenomas. AJNR. American journal of neuroradiology 15(8), 1591-1595 (1994).

9. Lienhardt, A., Grossman, A.B., Dacie, J.E., Evanson, J., Huebner, A., Afshar, F., Plowman, P.N., Besser, G.M., Savage, M.O.: Relative contributions of inferior petrosal sinus sampling and pituitary imaging in the investigation of children and adolescents with ACTH-dependent Cushing's syndrome. The 
Journal of clinical endocrinology and metabolism 86(12), 5711-5714 (2001). doi:10.1210/jcem.86.12.8086

10. Tabarin, A., Laurent, F., Catargi, B., Olivier-Puel, F., Lescene, R., Berge, J., Galli, F.S., Drouillard, J., Roger, P., Guerin, J.: Comparative evaluation of conventional and dynamic magnetic resonance imaging of the pituitary gland for the diagnosis of Cushing's disease. Clinical endocrinology 49(3), 293-300 (1998). doi:10.1046/j.1365-2265.1998.00541.x

11. Lefournier, V., Martinie, M., Vasdev, A., Bessou, P., Passagia, J.G., Labat-Moleur, F., Sturm, N., Bosson, J.L., Bachelot, I., Chabre, O.: Accuracy of bilateral inferior petrosal or cavernous sinuses sampling in predicting the lateralization of Cushing's disease pituitary microadenoma: influence of catheter position and anatomy of venous drainage. The Journal of clinical endocrinology and metabolism 88(1), 196-203 (2003). doi:10.1210/jc.2002-020374

12. Graham, K.E., Samuels, M.H., Nesbit, G.M., Cook, D.M., O'Neill, O.R., Barnwell, S.L., Loriaux, D.L.: Cavernous sinus sampling is highly accurate in distinguishing Cushing's disease from the ectopic adrenocorticotropin syndrome and in predicting intrapituitary tumor location. The Journal of clinical endocrinology and metabolism 84(5), 1602-1610 (1999). doi:10.1210/jcem.84.5.5654

13. Liu, Z., Zhang, X., Wang, Z., You, H., Li, M., Feng, F., Jin, Z.: High positive predictive value of the combined pituitary dynamic enhanced MRI and high-dose dexamethasone suppression tests in the diagnosis of Cushing's disease bypassing bilateral inferior petrosal sinus sampling. Scientific reports 10(1), 14694 (2020). doi:10.1038/s41598-020-71628-0

14. Aron, D.C., Raff, H., Findling, J.W.: Effectiveness versus efficacy: the limited value in clinical practice of high dose dexamethasone suppression testing in the differential diagnosis of adrenocorticotropindependent Cushing's syndrome. The Journal of clinical endocrinology and metabolism 82(6), 17801785 (1997). doi:10.1210/jcem.82.6.3991

15. Bertagna, X., Guignat, L., Groussin, L., Bertherat, J.: Cushing's disease. Best practice \& research. Clinical endocrinology \& metabolism 23(5), 607-623 (2009). doi:10.1016/j.beem.2009.06.001

16. Lonser, R.R., Nieman, L., Oldfield, E.H.: Cushing's disease: pathobiology, diagnosis, and management. Journal of neurosurgery 126(2), 404-417 (2017). doi:10.3171/2016.1.Jns152119

17. Zhang, W., Yu, Y., Tan, H., Wang, C., Li, J., An, Z., Liu, Y.: [Value of desmopressin stimulation test and high dose dexamethasone suppression testin the etiologic diagnosis of ACTH dependent Cushing's syndrome]. Zhonghua yi xue za zhi 96(11), 845-849 (2016). doi:10.3760/cma.j.issn.03762491.2016.11.004

18. Colao, A., Faggiano, A., Pivonello, R., Pecori Giraldi, F., Cavagnini, F., Lombardi, G.: Inferior petrosal sinus sampling in the differential diagnosis of Cushing's syndrome: results of an Italian multicenter study. European journal of endocrinology 144(5), 499-507 (2001). doi:10.1530/eje.0.1440499

19. Swearingen, B., Katznelson, L., Miller, K., Grinspoon, S., Waltman, A., Dorer, D.J., Klibanski, A., Biller, B.M.: Diagnostic errors after inferior petrosal sinus sampling. The Journal of clinical endocrinology and metabolism 89(8), 3752-3763 (2004). doi:10.1210/jc.2003-032249 
20. Grant, P., Dworakowska, D., Carroll, P.: Maximizing the accuracy of Inferior petrosal sinus sampling: validation of the use of Prolactin as a marker of pituitary venous effluent in the diagnosis of Cushing's disease. Clinical endocrinology 76(4), 555-559 (2012). doi:10.1111/j.13652265.2011.04257.x

21. Zampetti, B., Grossrubatscher, E., Dalino Ciaramella, P., Boccardi, E., Loli, P.: Bilateral inferior petrosal sinus sampling. Endocrine connections 5(4), R12-25 (2016). doi:10.1530/ec-16-0029

\section{Tables}

Table $1 \square$ Diagnostic confirmation and classification of 76 patients evaluated with pituitary dMRI and HDDST

\begin{tabular}{|c|c|c|c|}
\hline & \multicolumn{2}{|c|}{ Gold standard } & \multirow[t]{2}{*}{ Accuracy (\%) } \\
\hline & $\mathrm{CD}$ & EAS & \\
\hline \multicolumn{3}{|l|}{ Pituitary dMRI } & \\
\hline positive & 81 & 3 & \multirow[t]{2}{*}{80.7} \\
\hline Negative & 20 & 15 & \\
\hline \multicolumn{3}{|l|}{ HDDST } & \\
\hline Suppression(positive) & 71 & 4 & \multirow[t]{2}{*}{71.4} \\
\hline No suppression(negative) & 30 & 14 & \\
\hline \multicolumn{3}{|c|}{ Combined pituitary dMRI and HDDST } & \\
\hline Both positive & 60 & 1 & \multirow[t]{2}{*}{64.7} \\
\hline At least one negative & 41 & 17 & \\
\hline \multicolumn{4}{|c|}{ Combined pituitary dMRI and HDDST } \\
\hline At least one positive & 92 & 6 & \multirow[t]{2}{*}{87.4} \\
\hline Both negative & 9 & 12 & \\
\hline
\end{tabular}

CD: Cushing's disease, EAS: ectopic adrenocorticotropic hormone syndrome, dMRI: dynamic enhanced MRI, HDDST: high-dose dexamethasone suppression tests.

Gold standardøpathological examination

Both positive: positive findings in pituitary dMRI and cortisol levels suppressed by HDDST

Table 2: Diagnostic Performance of pituitary dMRI and HDDST in Detecting a Pituitary Source of ACTH 


\begin{tabular}{|c|c|c|c|c|c|c|}
\hline Test & $\begin{array}{c}\text { Sensitivity } \\
\text { (\%) }\end{array}$ & $\begin{array}{c}\text { Specificity } \\
\text { (\%) }\end{array}$ & $\begin{array}{c}\text { Positive } \\
\text { predictive } \\
\text { value (\%) }\end{array}$ & $\begin{array}{l}\text { Negative } \\
\text { predictive } \\
\text { value (\%) }\end{array}$ & $\begin{array}{c}\text { False } \\
\text { negative } \\
\text { ratio (\%) }\end{array}$ & $\begin{array}{c}\text { False } \\
\text { positive } \\
\text { ratio (\%) }\end{array}$ \\
\hline itary dMRI & 80.2 & 83.3 & 96.4 & 42.9 & 19.8 & 16.7 \\
\hline )ST & 70.3 & 77.8 & 94.7 & 31.8 & 29.7 & 22.2 \\
\hline $\begin{array}{l}\text { bined Pituitary dMRI } \\
\text { HDDST(both positive) }\end{array}$ & 59.4 & 94.4 & 98.4 & 29.3 & 40.6 & 5.6 \\
\hline $\begin{array}{l}\text { bined Pituitary dMRI } \\
\text { HDDST(either positive) }\end{array}$ & 91.1 & 66.7 & 93.9 & 57.1 & 8.9 & 33.3 \\
\hline
\end{tabular}

Figures 

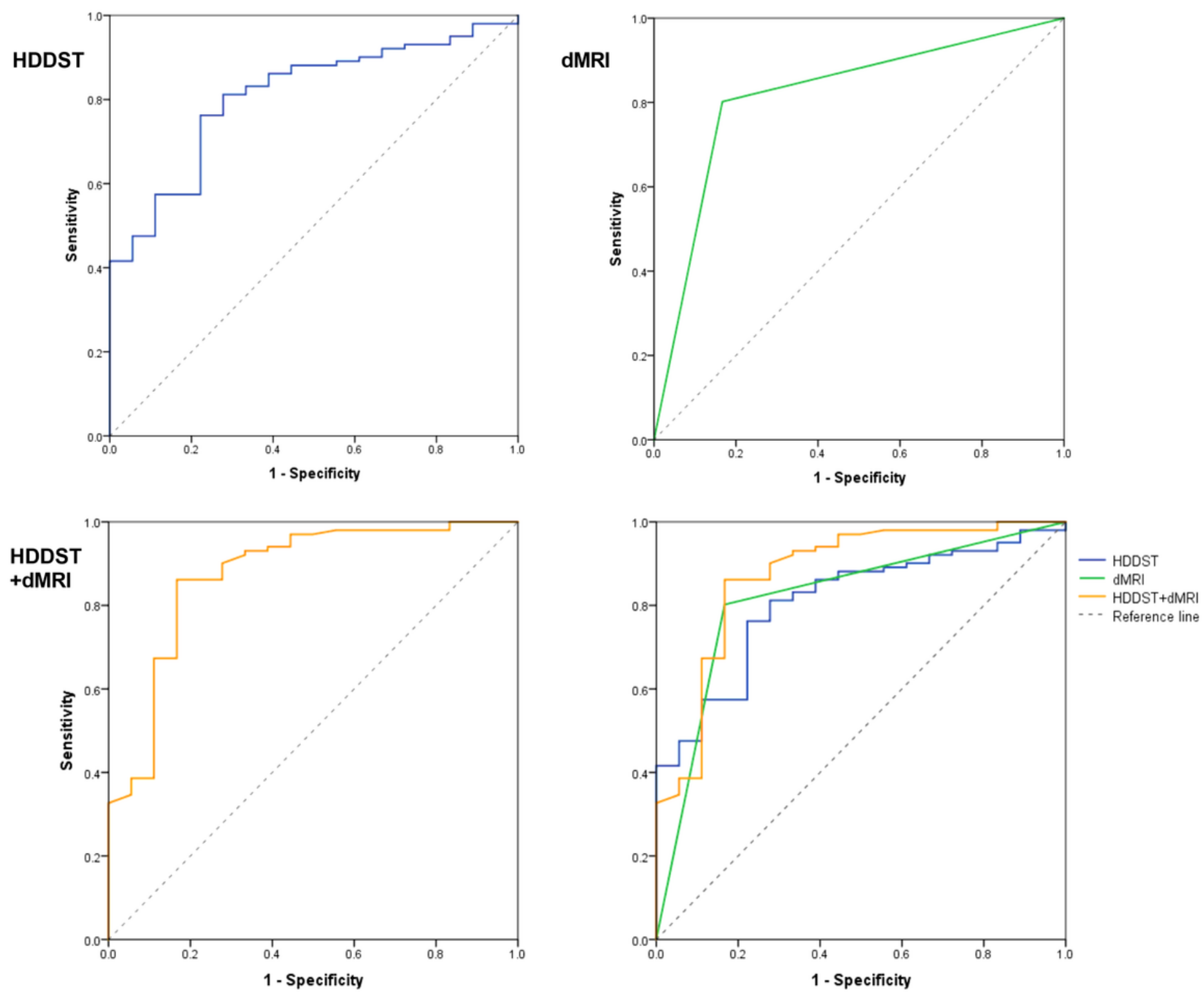

Figure 1

ROCs for the HDDST, dMRI, and the combined of HDDST and dMRI. 